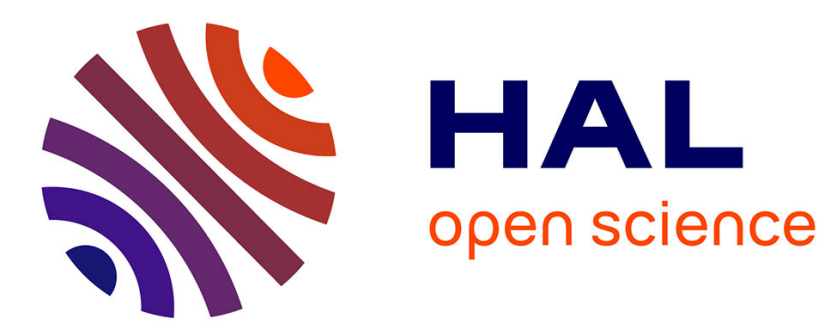

\title{
Dispositif de mesure et d'inscription automatiques de fréquences pour spectromètre hertzien à balayage lent
}

J.M. Lapaw, B. Macke

\section{To cite this version:}

J.M. Lapaw, B. Macke. Dispositif de mesure et d'inscription automatiques de fréquences pour spectromètre hertzien à balayage lent. Revue de Physique Appliquée, 1970, 5 (2), pp.351-356. 10.1051/rphysap:0197000502035100 . jpa-00243401

\section{HAL Id: jpa-00243401 https://hal.science/jpa-00243401}

Submitted on 1 Jan 1970

HAL is a multi-disciplinary open access archive for the deposit and dissemination of scientific research documents, whether they are published or not. The documents may come from teaching and research institutions in France or abroad, or from public or private research centers.
L'archive ouverte pluridisciplinaire HAL, est destinée au dépôt et à la diffusion de documents scientifiques de niveau recherche, publiés ou non, émanant des établissements d'enseignement et de recherche français ou étrangers, des laboratoires publics ou privés. 


\title{
DISPOSITIF DE MESURE ET D'INSCRIPTION AUTOMATIQUES DE FRÉQUENCES POUR SPECTROMẼTRE HERTZIEN A BALAYAGE LENT (*)
}

\author{
par J. M. LAPAUW et B. MACKE \\ Laboratoire de spectroscopie hertzienne, Faculté des Sciences de Lille, \\ Equipe de recherche associée au C. N. R. S.
}

(Reşu le 22 janvier 1970)

\begin{abstract}
Résumé. - Dans le cadre d'étude de double résonance en spectroscopie hertzienne des gaz à basse pression, nous avons été amené à développer un dispositif original de mesure et d'inscription des fréquences balayées utilisant les possibilités offertes par les compteurs numériques. Par sa souplesse d'emploi, ce dispositif semble utilisable dans des domaines très divers et en particulier dans les spectromètres à modulation d'absorption et à détection synchrone.
\end{abstract}

\begin{abstract}
In order to study double resonance effects in microwave spectroscopy of gases at low pressure, we built up a new device for the measurement and the transcription of the sweeped frequencies. Such a device uses the possibilities given by numerical counters and appears to be of a great versatility. The apparatus might be used in miscellanous types of experiments and namely with all the spectrometers based on the modulation of absorption and phase sensitive detection.
\end{abstract}

Introduction. - Les spectromètres hertziens les plus sensibles fonctionnent généralement sur le principe de la modulation d'absorption. En spectrométrie des gaz à basse pression, ceci est obtenu en modulant par tout ou rien le champ électrostatique dans les spectromètres Stark, la puissance de pompe dans les études de double résonance [2]. Le signal détecté est traité par le procédé d'amplification synchrone. La nécessité d'utiliser des constantes d'intégration assez grandes pour bénéficier d'un bon rapport signal/bruit, conduit généralement à choisir des balayages de fréquence lents et un enregistrement graphique des spectres. Les fluctuations de fréquence des sources (klystron ou carcinotron) sont corrigées en les asservissant en phase [1] [2] sur des pilotes radiofréquences. Grâce aux multiplications et aux transpositions de fréquence ainsi réalisées, le problème se réduit à la mesure d'une fréquence relativement basse, lentement évolutive. Cette fréquence (inférieure à $50 \mathrm{MHz}$ ) tombe dans le domaine d'emploi d'une grande variété de fréquencemètres. C'est pourquoi nous avons étudié une technique de mesure des fréquences avec impression des résultats par imprimante rapide.

I. Choix des procédés. - Le spectromètre particulier que nous utilisons [2] est destiné à l'étude des phénomènes de double résonance dans les gaz à basse

(*) Ce travail constitue le résumé d'une partie du mémoire [6] présenté par J. M. Lapauw au Conservatoire National des Arts et Métiers (Centre associé de Lille) pour obtenir le titre d'ingénieur C. N. A. M. pression [3]. La mesure des fréquences de pompe et de sonde nécessite celle de trois fréquences dont l'une est évolutive notée $F_{x}$ et les deux autres $F_{1}$ et $F_{2}$ fixes au cours d'un enregistrement. Les autres fréquences intervenant dans la chaîne de stabilisation sont fournies par des synthétiseurs (XUC Rohde et Schwarz et ND 30 MB Schomandl) ou des chaînes de multiplication alimentées par l'étalon $5 \mathrm{MHz}$ du laboratoire (General Radio 1115 B) et ne nécessitent pas de mesure.

En raison des exigences des servomécanismes d'asservissement en phase (Schomandl FDS 30) des oscillateurs de pompe et de sonde, qui n'acceptent pas de variation brutale de fréquence, la commande de fréquence s'effectue à l'aide d'une tension triangulaire. Celle-ci est appliquée également à la voie $\mathrm{X}$ de l'enregistreur (Hewlett Packard 7035 AM) ; elle est fournie par un générateur très basse fréquence (C. R. C. GB 860) qui permet éventuellement d'accélérer le mouvement pendant la phase de retour.

L'enregistrement de la figure d'absorption se faisant sur le front montant du signal triangulaire qui commande la source balayée en fréquence, nous avons choisi de mesurer la fréquence fixe $F_{1}$ avant, et l'autre fréquence fixe $F_{2}$ après l'enregistrement. Pendant l'enregistrement, la fréquence évolutive $F_{x}$ est mesurée à des intervalles de temps choisis, la plume de l'enregistreur étant levée un court instant de façon à marquer le signal d'un blanc au moment de la mesure (marquage en "modulation $Z$ ») [4] [5]. L'ensemble de ces mesures est réalisé suivant un cycle automatique 


\begin{tabular}{|c|c|c|c|c|c|c|}
\hline But & \multicolumn{2}{|c|}{ Fonction } & $\begin{array}{l}\text { Temps } \\
\text { de } \\
\text { comptage }\end{array}$ & $\begin{array}{l}\text { Cycle } \\
\text { de } \\
\text { comptage }\end{array}$ & $\begin{array}{c}\text { Levée de plume } \\
\text { de } \\
\text { l'enregistreur }\end{array}$ & $\begin{array}{l}\text { Commande d'impres- } \\
\text { sion des résultats } \\
\text { de mesure }\end{array}$ \\
\hline \multirow{3}{*}{$\begin{array}{l}\text { Réglages } \\
\text { préliminaires }\end{array}$} & \multicolumn{2}{|c|}{$\begin{array}{r}\text { Mesure de }\left|F_{x}\right| \\
\text { au hertz près }\end{array}$} & $\begin{array}{l}\text { Une } \\
\text { seconde }\end{array}$ & $\begin{array}{l}\text { toutes les deux se- } \\
\text { condes }\end{array}$ & $\begin{array}{l}\text { plume constam- } \\
\text { ment levée }\end{array}$ & $\begin{array}{l}\text { Pas d'impression. Affi- } \\
\text { chage visuel sur le } \\
\text { fréquencemètre. }\end{array}$ \\
\hline & \multicolumn{2}{|c|}{$\begin{array}{r}\text { Mesure de } F_{2} \\
\text { au hertz près }\end{array}$} & $\begin{array}{l}\text { Une } \\
\text { seconde }\end{array}$ & $\begin{array}{l}\text { toutes les deux se- } \\
\text { condes }\end{array}$ & $\begin{array}{l}\text { plume constam- } \\
\text { ment levée }\end{array}$ & $\begin{array}{l}\text { Pas d'impression. Affi- } \\
\text { chage visuel sur le } \\
\text { fréquencemètre. }\end{array}$ \\
\hline & \multicolumn{2}{|c|}{$\begin{array}{r}\text { Mesure de } F_{1} \\
\text { au hertz près }\end{array}$} & $\begin{array}{l}\text { Une } \\
\text { seconde }\end{array}$ & $\begin{array}{l}\text { toutes les deux se- } \\
\text { condes }\end{array}$ & $\begin{array}{l}\text { plume constam- } \\
\text { ment levée }\end{array}$ & $\begin{array}{l}\text { Pas d'impression. Affi- } \\
\text { chage visuel sur le } \\
\text { fréquencemètre. }\end{array}$ \\
\hline \multirow{3}{*}{$\begin{array}{l}\text { Enregistrement du } \\
\text { phénomène fonc- } \\
\text { tion de la fré- } \\
\text { quence } \\
\text { Inscription des ré- } \\
\text { sultats des mesu- } \\
\text { res permettant la } \\
\text { détermination de } \\
\text { cette fréquence en } \\
\text { chaque point de } \\
\text { l'enregistrement }\end{array}$} & & $F_{1}$ & $\begin{array}{l}\text { Une } \\
\text { seconde }\end{array}$ & $\begin{array}{l}\text { toutes les deux se- } \\
\text { condes }\end{array}$ & $\begin{array}{l}\text { plume constam- } \\
\text { ment levée }\end{array}$ & $\begin{array}{l}\text { Inscription des résul- } \\
\text { tats des mesures par } \\
\text { l'Imprimante. }\end{array}$ \\
\hline & $\begin{array}{l}\text { CYCLE } \\
\text { AUTO- } \\
\text { MATI- } \\
\text { QUE } \\
\end{array}$ & $\left|F_{x}\right|$ & $\begin{array}{l}\text { Un } 1 / 10 \mathrm{de} \\
\text { seconde }\end{array}$ & $\begin{array}{l}\text { variable suivant le } \\
\text { nombre de mar- } \\
\text { queurs désirés et } \\
\text { la durée du bala- } \\
\text { yage }\end{array}$ & $\begin{array}{l}\text { plume baissée } \\
\text { marqueurs en } \\
\text { «levée de plu- } \\
\text { me» pour cha- } \\
\text { que mesure }\end{array}$ & $\begin{array}{l}\text { Inscription des résul- } \\
\text { tats des mesures par } \\
\text { l'Imprimante. }\end{array}$ \\
\hline & & $F_{2}$ & $\begin{array}{l}\text { Une } \\
\text { seconde }\end{array}$ & $\begin{array}{l}\text { toutes les deux se- } \\
\text { condes }\end{array}$ & $\begin{array}{l}\text { plume constam- } \\
\text { ment levée }\end{array}$ & $\begin{array}{l}\text { Inscription des résul- } \\
\text { tats des mesures par } \\
\text { l'Imprimante. }\end{array}$ \\
\hline
\end{tabular}

Fig. 1. - Tableau des fonctions du D. M. I. A. F.

commandé par les impulsions délivrées par le générateur T. B. F. à chaque extrémum du signal triangulaire. Les résultats des mesures de $F_{1},\left|F_{x}\right|, F_{2}$ sont imprimés sur papier à l'aide d'une imprimante parallèle rapide (Sedelec TL 21).

Il est également prévu une possibilité de mesure classique de $F_{1}, F_{2},\left|F_{x}\right|$ sur le fréquencemètre sans impression des résultats, en vue de procéder aux réglages préalables à un enregistrement.

Pour réaliser toutes ces fonctions, nous avons utilisé un fréquencemètre (Rochar A 1439) dans des conditions spéciales. Pratiquement, nous avons exploité les possibilités de commandes extérieures du temps de comptage et de la remise à zéro du compteur.

1. Modification par commande extérieure du TEMPS DE COMPTAGE. - Le fréquencemètre utilisé fournit des signaux de fréquence $1 \mathrm{~Hz}, 10 \mathrm{~Hz}, 100 \mathrm{~Hz}$, avec la précision de l'étalon $5 \mathrm{MHz}$ qui pilote le compteur. En utilisant la fonction «période multipliée par dix », et en injectant sur une entrée auxiliaire un de ces signaux, on peut commander l'ouverture de la porte du compteur pour un temps égal à dix fois la période de ce signal. En sélectionnant la fréquence $(100 \mathrm{~Hz}$ ou $10 \mathrm{~Hz})$, on peut ainsi choisir le temps de comptage du fréquencemètre pour l'adapter à la définition nécessaire de la fréquence à mesurer, soit la dizaine de hertz ou le hertz suivant les cas.
2. REMISE A ZÉRO EXTÉRIEURE DU FRÉQUENCEMÈTRE. - Le fréquencemètre comporte une prise de remise à zéro extérieure des circuits d'automatisme et du compteur électronique. Le déclenchement d'un comptage est obtenu après avoir court-circuité cette prise pendant un temps supérieur à dix millisecondes. En maintenant le court-circuit, on empêche toute mesure et on prédétermine l'appareil pour une mesure qui s'effectuera quand on cessera le court-circuit. On peut ainsi, en envoyant un signal "cycle de comptage » sur l'entrée "remise à zéro » du fréquencemètre, choisir, suivant les fonctions à réaliser, le temps entre deux mesures du fréquencemètre.

Compte tenu des indications précédentes, des exigences sur la définition des fréquences, on peut fixer les fonctions à réaliser par notre dispositif. Ces fonctions sont résumées sur le tableau de la figure 1. Pour alléger l'écriture nous désignerons désormais notre dispositif par la notation D. M. I. A. F. (Dispositif de mesure et d'inscription automatiques de fréquences).

II. Réalisation des circuits. - II.1 SCHÉMA SYNOPTIQUE DU D. M. I. A. F. (Fig. 2). - Le dispositif comprend :

- un sélecteur de «fréquence» permettant de commuter l'entrée du fréquencemètre, par une commande électronique, sur l'une des voies fournissant le signal de fréquence $F_{1},\left|F_{x}\right|, F_{2}$; 


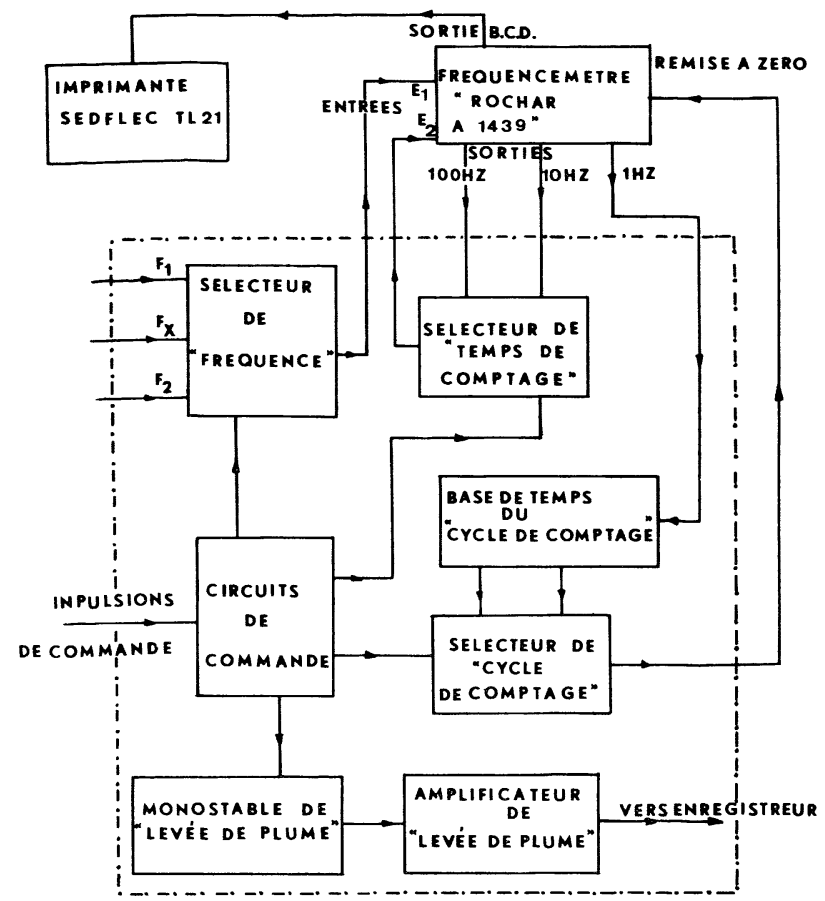

FIG. 2. - Schéma synoptique du dispositif de mesure et d'inscription automatiques de fréquences.

— un sélecteur de «temps de comptage» permettant de commuter l'entrée auxiliaire du fréquencemètre sur le circuit fournissant le signal de fréquence étalon $(10 \mathrm{~Hz}$ ou $100 \mathrm{~Hz})$ convenable ;

- un sélecteur de « cycle de comptage » permettant de commuter l'entrée « remise à zéro 》 du fréquencemètre sur le circuit fournissant le signal de déclenchement de la mesure convenable ;

- les circuits de commande, manuelle et électrique des divers sélecteurs ;

- une base de temps de "cycle de comptage", fournissant au sélecteur les deux signaux commandant les mesures du fréquencemètre :

- un signal rectangulaire de période deux secondes,

- un signal rectangulaire de période choisie entre deux et vingt secondes sur un commutateur de base de temps;

— un dispositif de «levée de plume» permettant de réaliser le marquage des courbes enregistrées.

L'alimentation du D. M. I. A. F. en + et -6 volts est fournie par le fréquencemètre.

II.2 Les « SÉLECTEURS DE voies» DU D. M. I. A. F. - II.2.1 Sélecteur de "fréquence ». - Les fréquences à mesurer sont toujours comprises entre $5 \mathrm{MHz}$ et $50 \mathrm{MHz}$. Comme le montre la figure 3, nous avons réalisé ce sélecteur à l'aide de trois interrupteurs à « effet de champ » $\left(T_{1}, T_{2}, T_{3}\right)$.

Ces interrupteurs diffèrent des interrupteurs à « effet de champ » habituels par le fait que la tension de commande est appliquée sur le drain et la source, la grille étant à la masse. La capacité drain source du transistor à effet de champ utilisée est inférieure à $0,01 \mathrm{pF}$.

Pour des signaux 0,3 volt efficace, ce qui est notre cas, l'atténuation en interrupteur fermé (commande à 0 volt), lorsque la sortie est fermée sur $50 \Omega$, est inférieure à cinq décibels, l'atténuation en interrupteur ouvert (commande à $+6 \mathrm{~V}$ ) lorsque la sortie est fermée sur $50 \Omega$, supérieure à 65 décibels.

Pour sélectionner une voie "fréquence », il suffit de porter la commande de son interrupteur à l'état 0 $(0$ volt $)$ et les deux autres à l'état 1 ( +6 volts).

Un des intérêts de ce sélecteur est qu'il ne demande aucune puissance de commande.

II.2.2 Sélecteur de «temps de comptage» et de "cycle de comptage ». - Lorsque l'on mesure une fréquence fixe $F_{1}, F_{2}$ on le fait toujours toutes les deux secondes et pour un temps de comptage de une seconde.

Lorsque l'on mesure la fréquence évolutive $\left|\left(F_{x}\right)\right|$, on fait cette mesure toutes les $x$ secondes (suivant le nombre de marqueurs désirés) et pour un temps de comptage de 0,1 seconde.

Les signaux assurant les fonctions «temps de comptage » et «cycle de comptage » sont commutés toujours en même temps, les deux inverseurs basculent donc toujours ensemble. C'est pourquoi leurs commandes sont réunies.

Comme le montre la figure 3 , nous avons réalisé ces inverseurs à l'aide de transistors ( $\mathrm{T} 8, \mathrm{~T} 9, \mathrm{~T} 10$ et $T$ 11). La commande se fait sur leur base, que l'on porte à un potentiel nul ou positif pour bloquer ou saturer des transistors suivant que l'on désire ou non laisser passer le signal de l'entrée correspondante.

II. 3 Circuits de Commande DU D. M. I. A. F. Ces circuits ont pour but: de commander les «sélecteurs de voies», et, pendant la mesure des fréquences fixes du cycle automatique, la levée de plume de l'enregistreur.

Les six tensions nécessaires à ces commandes sont fournies par les sorties de quatre bistables (B 20, B $20_{2}$, B $20_{3}$, B $20_{4}$ ). Ces bistables sont commandés, suivant la position du commutateur de fonction, soit par leur « remise à zéro », soit par les impulsions de commande issues du générateur T. B. F. et mises en forme par une bascule de Schmitt (D 20).

$\mathrm{Si}$ on place le commutateur «fonction» $\operatorname{sur}\left|F_{x}\right|, F_{2}$ ou $F_{1}$, quelles que soient les impulsions d'entrée, les bistables de commande sont placés dans un état préférentiel, par remise à zéro ou remise à un, de leur sortie S.

Les tensions obtenues pour chaque position $\left|F_{x}\right|, F_{2}$, $F_{1}$, du commutateur « fonction » sont celles qui placent les « sélecteurs de voies» dans les positions convenables pour réaliser les mesures de fréquences correspondantes, dans les conditions indiquées au tableau de la figure 1.

$\mathrm{Si}$ on place le commutateur fonction sur «cycle automatique », les circuits de commande ont été prédéterminés par le passage obligatoire du commutateur «fonction » sur la position $F_{1}$; c'est cette fréquence que l'on mesurera d'abord, puis, après la première impulsion de commande, ce sera $F_{x}$, puis, après la 


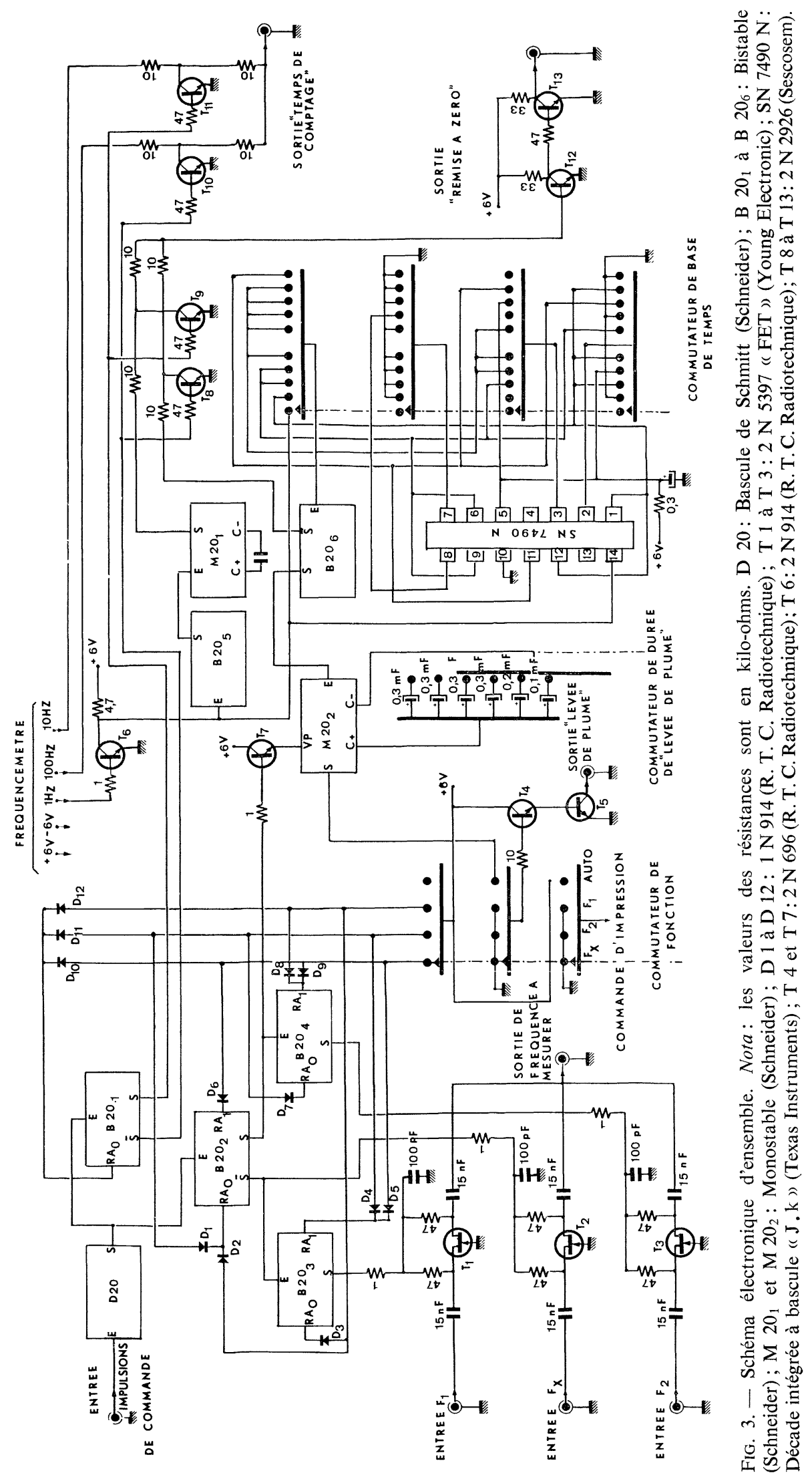


deuxième, ce sera $F_{2}$. Toutes ces mesures se font dans les conditions indiquées par le tableau de la figure 1 .

II. 4 BASE DE TEMPS DU D. M. I. A. F. - Cette base de temps doit fournir au sélecteur de «cycle de comptage » deux types de signaux :

a) Un signal de «cycle de comptage » pour la mesure des fréquences fixes. - Ce signal permet la remise à zéro du compteur toutes les deux secondes, pendant cent millisecondes. On peut ainsi faire effectuer par le fréquencemètre toutes les deux secondes une mesure d'une durée d'une seconde.

Il est obtenu à partir de fréquence étalon 1 hertz du fréquencemètre, par un bistable (B 20, ) suivi d'un monostable (M 20, $)$ dont le temps d'impulsion au niveau «zéro volt » est réglé à $100 \mathrm{~ms}$.

b) Un signal de "cycle de comptage » pour la mesure de la fréquence évolutive $\left|F_{x}\right|$. - Pour réaliser une échelle linéaire dans le temps d'une vingtaine de marqueurs en « levée de plume », donc d'une vingtaine de mesures correspondantes du fréquencemètre, pour des durées de balayage variant de 40 à 400 secondes, nous avons choisi de fournir des signaux de période $2,4,6$, $8,10,12,14,16,18,20$ secondes, suivant la position d'un commutateur de base de temps à dix positions.

Cela est obtenu à l'aide d'une décade intégrée à bascules 《 J.k » (SN $7490 \mathrm{~N})$, et d'un système de commutateur permettant de l'utiliser en diviseur par $1,2,3,4,5,6,7,8,9,10$ de la fréquence étalon $1 \mathrm{~Hz}$ $\mathrm{du}$ fréquencemètre (7).

En plaçant un bistable (B 20 $)$ à la sortie de cette décade, on obtient des signaux de période double et de rapport cyclique égal à l'unité. L'intérêt présenté par cette base de temps (en plus du fait que les marqueurs formeront une échelle linéaire dans le temps avec la précision de l'étalon du fréquencemètre), est que tous les signaux obtenus sont synchrones des signaux de fréquence étalon $100 \mathrm{~Hz}$ qui sont utilisés pour l'ouverture de la porte de comptage du fréquencemètre.

Cela signifie que le début effectif de la mesure de la fréquence variable, aura toujours lieu $10 \mathrm{~ms}$ après l'ordre de fin de remise à zéro, et de début de «levée de plume » de l'enregistreur.

L'inertie mécanique de la levée de plume de l'enregistreur étant du même ordre [4], on pourra admettre que le temps de mesure correspond bien au «blanc» $\mathrm{du}$ marqueur sur l'enregistrement.

II. 5 MONOSTABle ET AMPLIFICATEUR DE « LEVÉE DE PLUME » DU D. M. I. A. F. - Pour effectuer le marquage en «levée de plume » sur l'enregistrement, on remplace l'interrupteur mécanique par un interrupteur électronique, qui doit être ouvert pour que la plume soit levée, et fermé pour que la plume soit baissée.

Dans les positions $\left|F_{x}\right|, F_{2}, F_{1}$, du commutateur de fonction, la base du transistor $\mathrm{T}_{4}$ étant mise à la masse, l'interrupteur est ouvert, donc la plume constamment levée.

Dans la position «cycle automatique » la base de ce transistor est reliée à la sortie d'un monostable $\mathrm{M} 2 \mathrm{O}_{2}$ qui n'est lui-même alimenté que pendant la mesure de $\left|F_{x}\right|$. En effet, le signal de commande de $\mathrm{T}_{7}$ étant nul pendant la mesure de $F_{1}$ et de $F_{2}$, le monostable n'est pas alimenté, la sortie du monostable est à un potentiel nul, ce qui correspond à une levée de plume constante.

Pendant la mesure de $\left|F_{x}\right|$, le monostable est alimenté, et, en position de repos, la sortie est à un potentiel positif voisin de +6 volts. Le transistor de sortie est alors saturé, - ce qui correspond à un interrupteur fermé - la plume est baissée ; elle ne se lèvera qu'à chaque impulsion de commande arrivant au monostable et correspondant à une mesure de la fréquence $\left|F_{x}\right|$. Le temps d'impulsion à «zéro volt» du monostable, qui correspond à la durée de levée de plume, peut être choisi par modification de la capacité.

Un commutateur à six positions permet d'obtenir les «levées de plume» de 0,$1 ; 0,3 ; 0,6 ; 0,9 ; 1,2$; 1,5 seconde, pour adapter le temps de levée de plume à la vitesse de balayage utilisée.

III. Utilisation du D. M. I. A. F. - Pour effectuer les réglages préliminaires sur les chaînes de stabilisation de phase des sources hyperfréquences et sur le cadrage de son enregistrement, on se place sur une des positions $F_{1}, F_{2},\left|F_{x}\right|$ du commutateur «fonction $\gg$.

Sur toutes ces positions, la « levée de plume» est constante, l'enregistreur décrit donc «à blanc»la courbe à enregistrer. L'imprimante, d'autre part, ne reçoit pas du fréquencemètre le signal de transcription, donc n'imprime aucun résultat.

Quand les réglages sont terminés, l'expérimentateur peut passer sur cycle automatique pendant le temps de retour de la plume de l'enregistreur, c'est-à-dire le front descendant du signal «triangulaire » de commande (en général une dizaine de secondes avant le début du front montant de ce signal, pendant lequel a lieu l'enregistrement). Le dispositif étant alors (comme on l'a vu en II.3) prédéterminé, il effectuera quelques mesures de $F_{1}$. Puis à la première impulsion de commande, issue du générateur T. B. F., correspondant au début du front montant du signal «triangulaire » de commande, la plume de l'enregistreur descendra et à chaque marqueur en "levée de plume » correspondra une mesure de $\left|F_{x}\right|$. A la seconde impulsion du T. B. F., la plume se relèvera, le fréquencemètre effectuera alors quelques mesures de $F_{2}$, puis on arrêtera le cycle automatique en revenant sur une des positions $\left|F_{x}\right|, F_{1}, F_{2}$ du commutateur de fonction. Pendant toute la durée du cycle automatique, l'imprimante aura enregistré tous les résultats de mesure.

La figure 4 montre l'enregistrement d'un phénomène d'absorption sur lequel on a reporté les valeurs de fréquences mesurées pour chaque levée de plume, ces valeurs étant mesurées pendant un temps de comptage d'un dixième de seconde. Elles sont exprimées en 


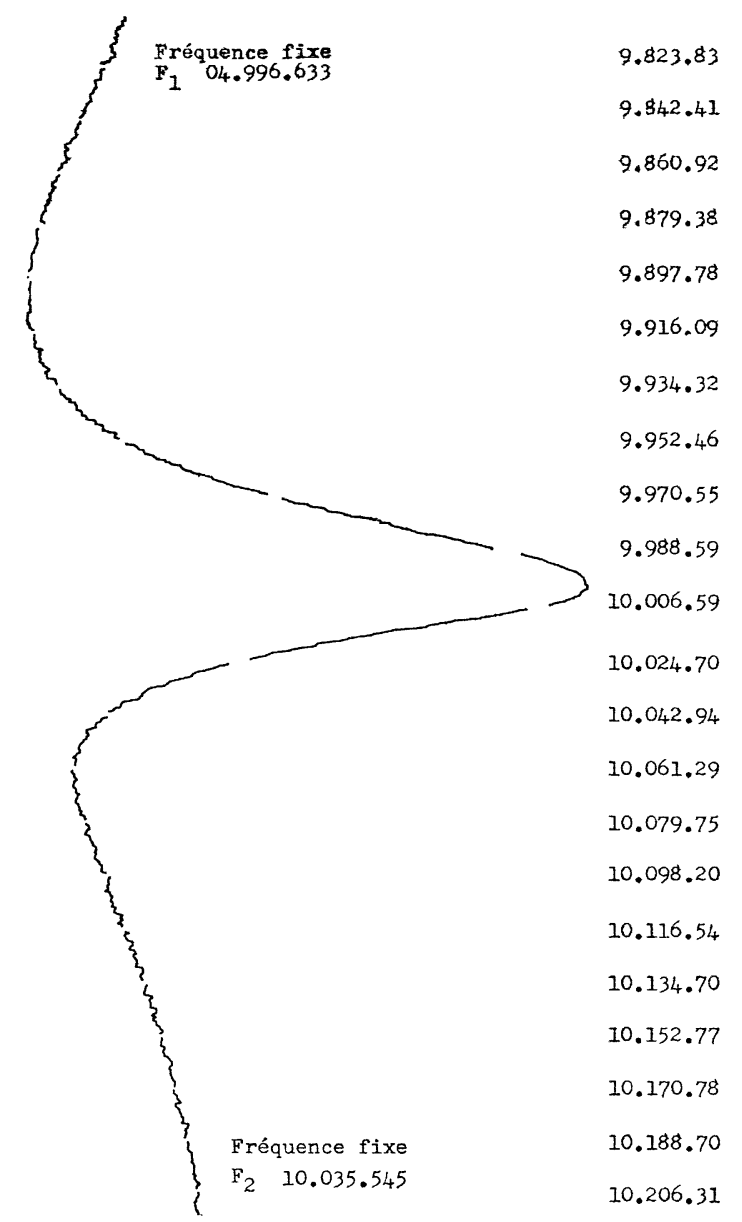

FIG, 4. - Enregistrement d'un phénomène d'absorption (balayage lent) dizaine de hertz. Les valeurs des deux fréquences fixes, mesurées avant et après l'enregistrement, ont été également reportées sur l'enregistrement; elles sont exprimées en hertz (temps de comptage d'une seconde).

Les autres données relatives à l'enregistrement sont :

- la durée de l'enregistrement : $3 \mathrm{mn} 50 \mathrm{~s}$,

- intervalle de temps entre deux mesures de la fréquence évolutive : $10 \mathrm{~s}$,

— durée des levées de plume : 0,3 s.

Conclusion. - Tel qu'il est réalisé, notre dispositif est adapté pour la mesure de fréquences inférieures à $50 \mathrm{MHz}$ et pour des durées de balayage comprises entre une et dix minutes.

Il est évident que ces limitations ne sont pas des limitations de principe, mais sont liées aux performances du compteur en ce qui concerne la limite supérieure de fréquence et à une réalisation particulière en ce qui concerne les durées de balayage.

A la différence des autres dispositifs de marquage en fréquence [4] des spectres hertziens, la méthode proposée n'implique aucune restriction ni sur la valeur centrale ni sur le domaine de variation de la fréquence balayée et permet de choisir la densité de marqueurs en fonction de la résolution requise.

Par ailleurs, notre dispositif est conçu en fonction des besoins des spectromètres à balayage de fréquences. Grâce aux convertisseurs tension/fréquence, on peut envisager l'extension de son emploi aux spectromètres à balayage de champs électrique [5] ou magnétique (R. P. E. et R. M. N.).

\section{Bibliographie}

[1] Journel (G.), Thèse, Lille, 1969 (C. N. R. S., no A 03417).

[2] Legrand (J.), Macke (B.), Messelyn (J.) et WerTheimer (R.), Rev. Physique Appl., 1968, 3, 237.

[3] Macke (B.), Messelyn (J.) et Wertheimer (R.), J. Physique, 1969, 30, 665.
[4] Legrand (J.), Macke (B.), Messelyn (J.) et WerTHeIMer (R.), Rev. Physique Appl., 1968, 3, 199.

[5] Burie (J.), Thèse de 3e Cycle, Lille, 1968.

[6] Lapauw (J. M.), Mémoire d'ingénieur C. N. A. M., Lille, 1969.

[7] KrausenER (J. M.), Electronique Industrielle, 1968, $117,627$. 